\title{
The Innovation Study of Management Theory
}

\author{
Fenghai ZHANG ${ }^{1,2}$ Tieshan HOU $^{1}$ Zhen WANG $^{3}$ \\ ${ }^{1}$ Department of Economics, Dalian University of Technology, Dalian China 116024 \\ ${ }^{2}$ School of Management and Social Science, Dalian Polytechnic University, Dalian \\ China 116034 \\ ${ }^{3}$ School of Economics and Management, Harbin Engineering University, Harbin China, \\ 150001
}

\begin{abstract}
Management in our country faces strong challenges at the turn of the century. By analyzing the hotspots disturbed in Chinese management field, a new idea is proposed in this paper. This idea showed that it is necessary to build a management theory to accommodate the requirements of times dynamically. Recognizing the effect of management, choosing the adequate management model, measuring the performance of administration and surveying the contribution of management in economic growth are also included. Meanwhile, the considerations and ways are given.
\end{abstract}

Keywords: Management Validity, Performance Evaluation, and Management Factor Contribution.

\section{Introduction}

With the coming of $21^{\text {st }}$ century, humans are stepping into a new development stage in which technology, economy and society are changing deeply and quickly. As a developing country, China faces both historical opportunities and severe challenges, which the latter exceeds the former. Men of insight feel the pressure and suffering. Relying on imitation of advanced techniques and experiences are hard to catch up with developed country; instead, it will reduce the action of "junior-develop superiority". Chances for labor-intensive industry, whose economical soar only depends on cheap labor force, will be smaller; the inequality of the old international economic order might stand out further. Then how to treat with the situation? The urgent affairs are innovating management theory, elevating management standard, and advancing with the time.

\section{Age Characteristic of Management}

Any country's flourish and society's progress always go with profound transform of management mode. For example, at the beginning of 20th century, US, the birthplace of modern management theory and management mode, finished the industrial revolution. Many of handworks were replaced by machine, which makes the work itself from complication and individual to easy and repetition. Without effective standard, with different approaches, workers completed the same work, and the decision-making was established just on intuition and foreboding. The situation did not change until Taylor invented a scientific theory, named Taylor management mode [1]. The theory was foremost spread and popularized in the US. Practice showed that it enhance the production rate to $200 \%$, which is the important reason that the enterprises who

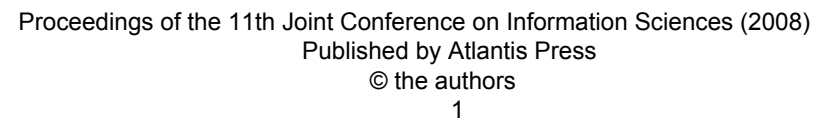


accepted the theory have gained more superiority than others who did not. And that established the US being the strongest economy country in the world.

However, Japan has been developing its economy invincibly since 1980's. In 1987, 50\% world semiconductor markets was taken over by Japan. While the US, the cradle of semiconductor industry, taken over only $25 \%$. The reason of why Japanese economy rapidly grew up is that the management mode they adopted meeting the demand of its native status. Japanese enterprises gained their cohesion by lifetime employment, seniority based employment, and labor union. Besides, the relation between the labor and the capital, and the sense of identification and adscription were integrative adequately. The human-oriented enterprise culture was the right weapon that Japan exceed the west developed country [2].

In fact, US researchers have long before begun to study on Japanese mode and its unique human-oriented management style, gained experiences both in culture and management. From culture to management, with the efforts of establishing a brand-new enterprise culture and innovating management theory in all aspects, US Companies recovered their vigour since 1990's, which makes huge increasing on GDP in the next decade.

It is obvious that a country's rise and fall is close related with the management mode and theory. In another words, the level of administration restricts the key factor of social evaluation. Famous management expert Drucker stated that: "developing countries drop behind not because of the laggardness of development, but the lower level of administration”, which points out the importance of administrating level enhancement. Also, Premier Zhu Rongji indicated before that implement the strategy of developing China through management. Although Chinese economy construction had made great progress in the practice of reinforcing management, there is still gap between the achievements we have got and the target we expected. To meet the requirement of advancing the level of administration, basically, we need to solve the drawbacks of current management theory.

\section{Management theory status and challenge}

It is necessary to resurvey the status and challenges that Chinese management field has to face, solving the management theory problem that baffled the enhancement of administration level.

\subsection{Debate in choosing management mode. Which one should be in the leading position, rational man- agement or irrational manage- ment?}

Currently, the relation between rational management and irrational management was unsuitable, which was accordingly considered by Chinese management field as the important reason of management tropism warp. Different attitudes exist on how to deal with the relation correctly. Some researchers hold that the transformation from "hard" management, which based on rational object-oriented idea, to the "soft" one, which based on irrational human-centered idea, should be carried out, where the latter was set in the leading position. Others put forward that the "hard" one should be dominant in the coexistence of rational and irrational management [3].

\subsection{The diversity of performance evaluation system, based on out- put or behavior?}

Performance evaluation (PE), the important foundation of management mode, has such two opposite theoretical system "that are far from coming to an agreement” [4], one is based on output, and the

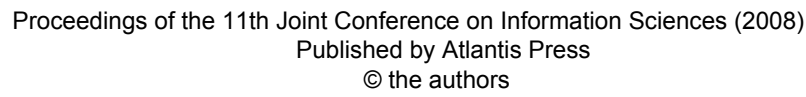


other is on behavior. The output based PE incline to denote evaluating unit output record in a given period. Data Embedding Analysis (DEA) method, which A. Charnes established in 1978, could be the representative work in output based PE recent years [5]. Based on DEA, many researchers in economical field study $\mathrm{PE}$. Significant research fruit obtained. The behavior based $\mathrm{PE}$ regards the output record as a phenomenon that behavior factors resulted in. These factors, reflecting the essence of performance, include diathesis, ability, efficiency endeavor degree ... etc. Recent years, enterprise culture theory and core competitiveness theory give a representation on the behaviorbased work. As market competition is impetuous increasingly, people recognized that it is important to consider the economic benefit from the point of view in long-term organizational development. Harvard School, leading by Robert K., brings forward a strategic management idea that people think much of it [6]. Strategic management emphasizes the function of non-finance performance, which makes use of the behavior factors above.

However, the bifurcation in understanding PE and PE theory system drawbacks deeply impact on the improvement of Chinese industries' performance. Facing to current impetuous economy compete, we have no time to delay to establish a nicety PE theory system.

\subsection{Is management effective? How to measure the management effi- ciency?}

At present, build a management validity theory and measure approach is an urgent task. But we have to solve some difficult problems first, such as how to define the concept of management validity, how to measure it ... etc. Objective condition in performance evaluation is the main point of analysis the management efficiency. The diversity of objective conditions influences the whole evaluation unit level.
The diversity was reflected in difference of internal factors, such as capital, technique and labor. On the other hand it reflected in difference of external environmental factors. It could be discovered from literature that, in China, companies that located in littoral with good fundamental condition usually showed better performance than northwest enterprise with worse condition. Whereas, it not means that the managers in northwest have lower diathesis, ability, and efficiency endeavor degree than those in littoral. Although researchers had recognized these problems, there was no appropriate way given to deal with. It is what we exert ourselves to settle.

\subsection{How to measure management fac- tor contribution in economic growth? How to distribute ac- cording to contribution?}

As the most important factor involving in the course of produce, management plays a decisive role in economic growth. Quantitatively measure the effect of management is advantaged to build a new distribution system, to carry out the sprit of distribution according to contribution. To measure the management factor contribution in economic growth has become another point in management field.

Equitableness and efficiency are involved in the principle of income distribution; as a consequence, it was one of big social economy problem all through the ages. Corporation could obtain super profit by management power. On protecting manager's legal rights and interests, thus, become an essential issue. In that, if the rights and interests were not treated with properly and duly, either their enthusiasm will be damaged or it might cause enterpriser corruption. Implementing distribution according to contribution could fully exert enterpriser's human capital, which is in fact a rare resource, could overcome the capital's Cannikin Effect [7]. Recently, establishing the distribution

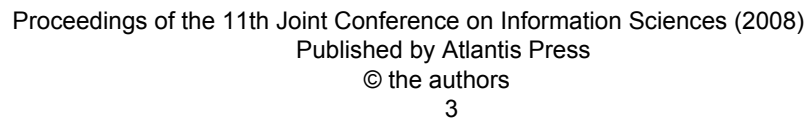


principle that introduce contributing factor, namely labor, capital, technique and management ...etc., are proposed. The principle is the key measurement in fulfilling the strategy of developing China through management. However, within various input of these factors, measuring the contribution in economic growth, implementing distribution according to the contribution are always the project people explore continuously in theory but far from figure out.

\section{Build a management theory meet- ing the demand of times ever changeably}

No innovation management theory, no innovation management action. Management theory must evaluate continuously along with times. The imitation and follow of foreign management mode has less help on break away from lag. To make Chinese catch up with developed countries directly, none but original innovation putting forward in light of the Chinese situation, including social culture environment and management foundation.

Currently, the common limitation of management theories is observing management function and evaluating its performance in a static way. The function was considered as the performance that effectively realized the management targets, and by reviewing the achievement of these static targets, evaluated the performance. It is difficult to adopt our country to the impetuosity environment full of international competition, to the "Human-centered" tenet in knowledgebased economy. As the matter of fact, the difficulty results from the static study of management theory. In the phylogeny of management science, whatever the theory and approach influential are, all combined with era closely. Therefore management target and strategy should be, from dynamic changing point of view, building a management theory meeting the demand of times. The followings are the way to solve the hotspot problem that authors gave:

\subsection{Unify performance evaluation systems}

Study the two PE systems based on output and behavior displayed that the output-based PE has an intuition expression and is easy to give a quantity research on it. Nevertheless, it is always influence by the diversity of objective conditions of evaluation unit. Inevitably the estimation merely shows the strength of evaluation unit and hardly reflects efficiency endeavor degree. Without question it is lack of fairness. In the case, people are easy to draw a conclusion only from the conditions, less from subjective efforts about the low efficiency of their behaviors. The inspire action of output-based PE would be limited. Different from it, the PE based on behavior, which mastered the essence of PE better, is hard to quantitate. Expertgrading method could hardly be reasonable because much artificial ingredients are involved, meanwhile negative influence would produce. To sum up, dividing the PE into output-based and behaviorbased tends to cause chaos.

In our opinion, there is no abstract "behavior factor". The integration of factors related to behavior, including manager's diathesis, ability, and efficiency endeavor degree, cannot but put into effect on the output strength variety of evaluation unit. Contrariwise, using the dynamic variety of evaluation could also measure the relative synthesis effect caused by the above factors (the measuring way see also reference [8]). The measurement shows the whole status of evaluation unit, as well as a certain lay. Besides, the measurement does not deny the evaluating function based on output record, but explores the deep-seated information provided when the strength change dynamically, calculates the relative effect of behavior fac-

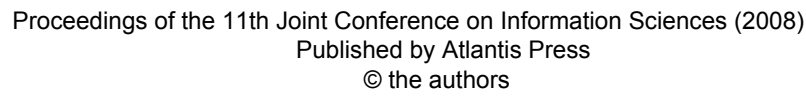


tors in a synthetic way. Hence, it is possible to combine the two PE systems.

\subsection{Tropism of management mode}

In rational management aspect, domestic enterprises, which shaped in Chinese traditional culture that possess irrational management features, have severe defect. These defects weaken the management base itself and block the development and enhancement of management level. Under condition of the planned economy mode formed after 1949, peoplecontrolled rather than law-rule was taken granted. Looking down on the system establishment of standardization, hardly complying with laws, scarcely following the rules caused a low degree of specialization and standardization. Many lessons convinced that, at present, the rational management should be in dominant but the irrational mode should not be ignored. With the emerging of knowledge-based economy age, the proportion of the irrational mode is increasing that make demands on giving attention to both management mode, when we commit ourselves to the rational mode.

\subsection{Management validity measure- ment}

Management exerts human's effect, taps the latent power as much as possible. Management validity is a behavior characteristic that eliminates the influence of the diversity in objective condition and reflects the benefit produced by management. In the proposed idea, the influence on PE caused by objective condition diversity from different evaluation unit is avoided. The whole strength of a unit is considered as the summation of all diathesis it has, including fundamental conditions, work behavior and management capabilities ... etc. Managers’ efficiency endeavor degree and capability would also act on the strength. So, their efficiency endeavor degree could be measured by the dynamic changing of the strength, which makes the PE much more scientific. (The measuring way sees also reference [8]). It is obvious that the $\mathrm{PE}$ method based on validity could survey the managers' efficiency endeavor degree objectively and justly, which conduce to analyze the reason why manager himself has the low efficiency about the behavior his own.

\subsection{The management factor contribu- tion measurement}

According to the economic growth theory, management factors contribution is the contribution rate acting in economic growth. For one thing we have to solve how to measure the rate. It is significant both in theory and in practice that building a scientific and reasonable calculates method.

The precondition of measuring the contribution quantificationally is confirm in advance what point of view should be taken when understand the function of management and what kind of method should be taken in calculation contribution rate. At this point, we bring forward the following two opinions:

(1) Management function

Management has vivid age characteristic. People have different understanding of management function in different times. But they did not pay any attention to understanding it from a dynamic angle of view. Nowadays, only if we take management as an organizational behavior impelled benefit could Chinese management adopt itself to the singularly impetuosity international competitions It accord with the economic growth theory, help to implement the innovation of management theory. Meanwhile, in light of variety benefit, the method of measuring the contribution could be built.

(2) Management factor contribution

Changing with time, benefit in equivalent benefit facet production function

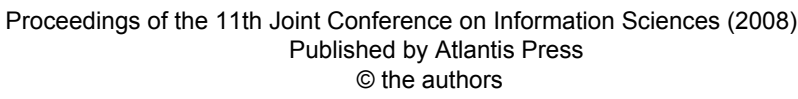


could describe management factor contribution. Same as the rise rate equation deduced by Robert M. Solow, we could get a corresponding rise rate function in equivalent benefit facet production function. Furthermore, using evaluation unit rise rate function, we could get the measure approach by which the management factor contribution in economic growth could be calculated ${ }^{[9]}$.

Now we need to understand the function of management dynamically, measuring the management performance, calculating the factor's contribution in economic growth. All these are for one target that making the management validity and its contribution rate as a core, centering on it, forms an effective management theory system, which could accord with the situation of our country and could meet the demand of enhancing the management level, eventually, put the system into practice, impel the productivity developing, advance with time.

\section{Conclusions}

Facing the unavoidable management revolution, we believe that the key is to bring forth new ideas about management theory continuously, to study the everchangeable management function, to endow management new meaning and take it as an important factor making the benefit increased. It is in favor of uniting the two management modes, eliminating the divergence of two PE systems, in favor of dispelling the difference of objective condition, reflecting the effective degree of manager's subjective endeavor, in favor of calculating the management factor's contribution in economic growth by equivalent benefit facet production func- tion, building a management theory that meet demands.

\section{References}

[1] Gary Dessler. Human Resource Management. Prentice-Hall International, Inc. 1997.256-287.

[2] SHI Yuan. Subtle influence on Culture Mode and incessant change of management mode. Chinese and Foreign Management Review, 2001, (2): 22-24.

[3] NIE ZhengAn. The Warp and Adaptation of Chinese Enterprises Tropism of management mode. Economic Review. 2000, (2): 119-122.

[4] Richard S.Williams. Performance Management. International Thomson Business Press, 1999.140-151.

[5] R.S.Kaplan, D.P.Norton. The Balanced Scorecard: Translating Strategy into Action. Harvard Business School Press.1996.135-146

[6] A.Charnes, W.W.Cooper, E.Rodes. Measuring The Efficiency of Decision Making Units. European Journal of Operational Research, 1978, (2): 429-444

[7] PI JianCai. Management is also the distribution factor. International Finance News. Nov $13^{\text {th }} 2002$

[8] WANG Zhen, FENG Ying-Jun, MENG Yan. Job\&CompetencyBased Dynamic Human Resource Management Mode. China Soft Science, 2003, (9): 69-73

[9] FENG Ying-Jun, MA Kui-Dong, SUN Jian-Fei. A Measurement Method of Management Contribution Rate in Economic Growth. Quantitative \& Technical Economic, 2003, (3): 49-53 